\title{
Wilms' tumor 1 gene (WT1) is overexpressed and provides an oncogenic function in pediatric nephroblastomas harboring the wild-type WT1
}

\author{
SURASAK SANGKHATHAT ${ }^{1,2}$, SAMORNMAS KANNGURN ${ }^{1,3}$, WELAWEE CHAIYAPAN ${ }^{1,2}$, \\ PODCHANAPORN GRIDIST ${ }^{1,4}$ and WANWISA MANEECHAY ${ }^{5}$ \\ ${ }^{1}$ Tumor Biology Research Unit; Departments of ${ }^{2}$ Surgery, ${ }^{3}$ Pathology, and ${ }^{4}$ Biomedical Science; \\ ${ }^{5}$ Molecular Biology Laboratory, Faculty of Medicine, Prince of Songkla University, Songkhla 90110, Thailand
}

Received March 5, 2010; Accepted May 20, 2010

DOI: 10.3892/ol_00000109

\begin{abstract}
Wilms' tumor 1 gene (WT1) is known to be a tumor suppressor gene in the subset of nephroblastomas that harbors WT1 mutations. However, its role in nephroblastomas without mutations remains unclear. This study aimed to evaluate the expression of $W T 1$ and its potential oncogenic role in pediatric nephroblastoma with wild-type WT1. A total of 24 nephroblastomas were studied for WT1 mRNA expression by quantitative reverse-transcription polymerase chain reaction. The expression levels were compared between nephro-blastomas with and without WT1 mutations, as well as to normal kidney tissue, other pediatric renal tumors and neuroblastomas. Immunohistochemistry was used to evaluate expression patterns at the tissue level. Post-transcriptional inhibition of WT1 was performed in primary cultures of wildtype nephroblastoma using WT1 siRNA. The average WT1 expression level in nephroblastoma tissue was significantly higher than that in normal kidney tissue and neuroblastomas. Expression at the mRNA level was not different between nephroblastomas with WT1 mutations (4 cases) and those with wild-type WT1 (20 cases). However, while WT1 immunoreactivity was positive in all of the nephroblastoma components in the tumors with wild-type $W T 1$, the protein expression was weaker and limited to stromal components in the tumors with mutated WT1, where it co-localized with $\beta$-catenin nuclear accumulation. The post-transcriptional inhibition of WTI resulted in growth retardation and a significantly increased apoptotic fraction. Our study found overexpression of the WT1 gene in pediatric nephroblastomas with wild-type WT1.
\end{abstract}

Correspondence to: Dr Surasak Sangkhathat, Tumor Biology Research Unit, Faculty of Medicine, Prince of Songkla University, Hat Yai, Songkhla 90110, Thailand

E-mail: surasak.sa@psu.ac.th

Key words: nephroblastoma, Wilms' tumor, Wilms' tumor 1 gene, $\beta$-catenin
Moreover, the study suggests an oncogenic role of WT1 in this tumor subset.

\section{Introduction}

Nephroblastoma (Wilms' tumor) is a common pediatric embryonal tumor that occurs as a result of abnormal cellular differentiation during organogenesis. Nephroblastomas typically exhibit triphasic histological components, consisting of blastema, epithelia and stroma (1). According to molecular pathology, tumors are now subcategorized into 2 subclasses by Wilms' tumor 1 gene (WT1) mutation status (2). The WT1mutated subclass usually contains a concomitant $\beta$-catenin mutation while the WT1 wild-type subclass harbors mutations of a novel candidate gene, WTX, in varying frequencies $(3,4)$. While $W T 1$ is known to be a classic tumor suppressor in the WT1-mutated subclass, however, its role is unclear in the WT1 wild-type subclass as it appears to possess a different tumorigenesis pathway (2).

WT1 encodes a zinc-finger protein that functions as a transcription factor involved in cellular growth and differentiation (5). A physiological role of WT1 in renal development during embryogenesis was confirmed by a previous study (6). The gene was originally described as a tumor suppressor gene that is involved in the tumorigenesis of nephroblastoma. However, only $10-15 \%$ of nephroblastomas harbor WT1 mutations, and growing evidence suggests that nephroblastomas with mutations of WT1 possess a separate molecular pathogenesis to nephroblastomas without mutations (2). Thus, the role of WT1 should be re-assessed in the WT1 wild-type subclass. WT1 plays an oncogenic role in adult renal cell carcinoma and various other human neoplasms, particularly leukemia, breast and prostate cancers. These factors suggest a possible oncogenic role for WT1 in pediatric nephroblastomas.

This study aimed to observe the expression of WT1 in pediatric nephroblastomas. The expression levels were analyzed with regard to WT1 mutation status and compared to other pediatric renal tumors and neuroblastomas. Furthermore, WT1 RNA interference was performed in a primary culture model. The negative effects thereof were also demonstrated in tumor growth, which was related to increased apoptosis. 


\section{Materials and methods}

Tissue samples and nucleic acid extraction. Frozen tumor tissue from 24 cases of nephroblastoma and their normal kidney counterparts, 3 cases of other pediatric renal tumors and 10 cases of neuroblastomas were used in the mutation and RNA-based expression studies. The 'other renal tumor' group consisted of 2 cases of clear cell sarcoma of the kidney and a case of pediatric renal cell carcinoma that harbored PRCC-TFE fusion. Sample collection and use of clinical data were performed under the guidelines of the Institutional Research Ethics Committee (Project 50/368-023) of the Faculty of Medicine, Prince of Songkla University. DNA extraction from the samples was carried out using a Genomic DNA mini kit (Geneaid, Taiwan) following the manufacturer's suggested protocol. Total RNA was extracted using an RNAeasy extraction kit (Qiagen, Inc.).

For the immunohistochemical study, formalin-fixed, paraffin-embedded archival tissue samples from the 24 cases of nephroblastoma were used. Clinical data from the same series of patients were published in a previous study (7). Briefly, the series included 2 patients who presented with a partial spectrum of WAGR (Wilms' tumor, aniridia, genitourinary tract anomalies and retardation) association and 2 patients with bilateral tumors. None of the patients had a family history of renal tumors.

WT1 and $\beta$-catenin mutation study. The full coding sequence of WTI was studied in each nephroblastoma sample by polymerase chain reaction (PCR) and the direct nucleotide sequencing method. The primers and PCR conditions for WTI were identical to a previous study (3), with some modifications. The mutation study of $\beta$-catenin spanned exon 3 , which encodes its phosphorylation sites. Sequencing was performed in both directions.

WT1 and $\beta$-catenin expression study. The relative expression of $W T 1$ and $\beta$-catenin at the mRNA level was studied with quantitative real-time reverse transcription PCR (qRT-PCR). In brief, cDNA was constructed from $1 \mu \mathrm{g}$ of RNA using an Omniscript reverse transcription kit (Qiagen, Inc.) following the manufacturer's instructions. qRT-PCR was conducted with an ABI7300 model real-time PCR machine (Applied Biosystems, Inc.) and a QuantiTech Probe RT-PCR kit was used (Qiagen, Inc.). Expression of WTI in terms of copy numbers was normalized with $10^{-5}$ copies of GAPDH and was presented logarithmically. The relative expression of $\beta$-catenin was also performed in a similar manner. Details of the primers and Taq Man probes were previously published $(8,9)$.

The expression and localization of WT1 at the protein level were studied in 24 clinical nephroblastoma tumor tissue samples using immunohistochemistry. Hematoxylin and eosin-stained slides from the case records were selected by a pathologist for immunohistochemical study. Sections (3- $\mu \mathrm{m})$ were cut, deparaffinized and rehydrated from formalin-fixed, paraffin-embedded tissue. A WT1 monoclonal antibody (1:500; Dako, Inc.) was used as the primary antibody. The staining followed the manufacturer's suggested protocol for the Dako EnVision+ System (Dako, Inc.). Briefly, antigen retrieval was performed in a microwave oven using Tris-EDTA buffer.
Endogenous peroxidase activity was blocked with $0.03 \%$ hydrogen peroxide containing sodium azide. Slides were incubated with non-immune serum for $30 \mathrm{~min}$ and then with the primary antibody for $120 \mathrm{~min}$ in a moist chamber, followed by an additional 30-min incubation with peroxidase-labeled polymer conjugated to goat anti-mouse immunoglobulins. Color was developed through a liquid 3,3'-diaminobenzidine chromogen solution. Light counterstaining was carried out with hematoxylin. $\beta$-catenin immunostaining was performed using the same protocol described in a previous publication (10). The immunohistological staining in this study was performed by one technician.

Primary culture of nephroblastoma. Primary nephroblastoma cells were derived from the ascites of a patient with recurrent nephroblastoma. The primary tumor of this patient was in stage 3 (gross tumor rupture prior to surgery), showed favorable histology and harbored wild-type WT1 and $\beta$-catenin. Following centrifugation of the ascites, the pellet was washed 3 times with phosphate-buffered saline (PBS). The cells were resuspended with RPMI-1640 (Invitrogen, Inc.) supplemented with $20 \%$ fetal bovine serum (FBS) (Gibco BRL), $1 \%$ penicillin/streptomycin and $0.5 \%$ glutamine. Cells were grown in $60-\mathrm{mm}$ petri dishes and incubated in a $5 \% \mathrm{CO}_{2}$ at $37^{\circ} \mathrm{C}$. The first medium change was carried out on the second day when the cells began to attach properly. The medium was changed every 3 days until the culture reached $80 \%$ confluence. After the two passages, an aliquot of cells was cryopreserved in 90\% FBS/10\% dimethyl sulfoxide and stored in liquid nitrogen. All experiments were performed on the fourth passage. Further subcultures showed that the cells ceased their proliferative ability at the fourteenth passage at 4 months of continuous culture.

WT1 RNA interference and Annexin-V apoptosis assay. The siRNAs against WT1 $\left(\right.$ siRNA $\left._{\mathrm{WT1}}\right)$ used in this study were presented in a previous publication by our group (11). Briefly, siRNA $_{\mathrm{WT} 1}$ was a combination of three 25-nt siRNA duplexes targeting different non-overlapping regions of WT1 mRNA on exons 7 and 8.

One day prior to transfection, a total of $1.5 \times 10^{5}$ cells were seeded in each well of a 24 -well plate. The cells were transfected with siRNA $\mathrm{wTI}_{\mathrm{WT}}$ at a final concentration of $100 \mathrm{nM}$ using Fugene6-HD (Roche, Inc.) as a transfection agent. The transfection agent plus a non-specific sequence (Invitrogen, Inc.) was used as a mock control. Cells were harvested at 48 and $72 \mathrm{~h}$ after transfection to check the expression of WT1 mRNA. The expression was semi-quantitatively determined using the RT-PCR method, using 28 cycles of reaction and the same primer set as WT1 qRT-PCR. The cell survival assay after WTI inhibition was performed in a 24-well plate format. The cells were plated at a near-confluence density of $5 \times 10^{4}$ cells/well. Following siRNA $A_{\mathrm{WT} 1}$ transfection, the number of cells in each group was determined by counting under a hemocytometer using the trypan blue exclusion technique.

The apoptosis experiment was performed in a $60-\mathrm{mm}$ disc format. Cells were plated in three discs one night prior to transfection. siRNA $\mathrm{w}_{\mathrm{WT1}}$ and the non-specific sequence were transfected at the same concentration as the experiment in the 6-well plate format. The cells were trypsinized and harvested at 
Table I. WT1 and $\beta$-catenin genotypes and the WT1 immunoreactivity pattern of 4 nephroblastoma cases with WT1 mutations.

\begin{tabular}{lllll}
\hline Code & Clinical remarks & \multicolumn{1}{c}{ WT1 mutation } & $\beta$-catenin mutation & WT1 immunohistochemistry \\
\hline WT8 & WAGR association & Exon 8 CGA413TGA (Arg413stop) & TCT45TAT ser45tyr & Stroma (+) \\
WT13 & Bilateral disease & Exon 8 CGA413TGA (Arg413stop) & - & Stroma (+) \\
WT20 & - & Exon 9 CGG445TGG (Arg445Trp) & TCT45TGT ser45cys & Blastema (++) \\
WT24 & WAGR association & Exon 7 151-152 (ins 32 bp) & TCT45TTT ser45phe & Stroma (+) \\
\hline
\end{tabular}

WAGR, Wilms' tumor, aniridia, genitourinary tract anomalies and retardation; ins, insert.

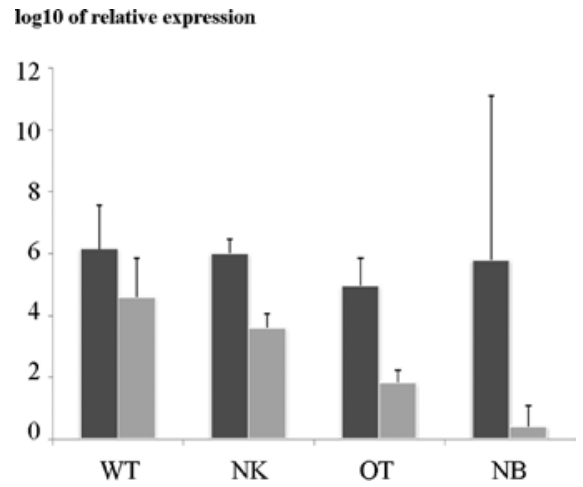

Figure 1. Relative expression of WT1 (grey bar) and $\beta$-catenin (dark bar) in pediatric nephroblastomas (WT), normal kidney tissue (NK), other pediatric renal tumors (OT) and neuroblastoma (NB). Copy numbers of WT1 and $\beta$-catenin were normalized by $10^{-5}$ copies of GAPDH and displayed in logarithmic scale.

$72 \mathrm{~h}$ of transfection. The apoptosis assay used the double staining of Annexin- $\mathrm{V}$, the propidium iodide method and flow cytometry on the Becton-Dickinson FACScan platform. Flow cytometric analysis was conducted using the CellQuest program.

\section{Results}

Mutations of WT1 and $\beta$-catenin in pediatric nephroblastoma. Among the 24 nephroblastomas examined, WT1 mutations were detected in 4 cases (Table I). Two of these cases had associated WAGR syndrome and 1 case had bilateral tumors. Of the 4 tumors with a WT1 mutation, concomitant mutations at the $\beta$-catenin codon 45 were detected in 3 tumors. Except for WT1 mutations detected in the normal tissue of 2 WAGR cases, all other mutations were somatic.

Relative overexpression of WT1 in pediatric nephroblastoma. In the qRT-PCR study, the relative expression of WT1 in the nephroblastomas was significantly higher than that in the control renal tissue and the other renal tumors. The expression of WT1 in the nephroblastomas and other pediatric renal tumors was higher than that in the neuroblastoma tissues (Fig. 1). No significant difference was noted in the relative expression of $\beta$-catenin among the nephroblastomas and other types of tissue studied. The relative expression of WT1 in the nephroblastomas harboring a WT1 mutation was not different from wild-type tumors (data not shown).

Characteristic patterns of WT1 immunohistochemistry in nephroblastoma. WT1 immunoreactivity was detected in varying
WT1-wild-type

A

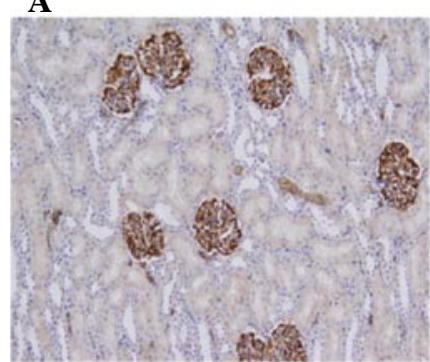

\section{B}

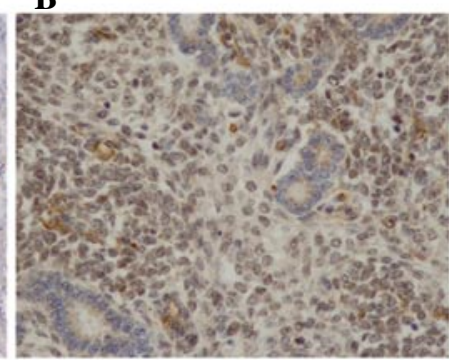

C

D
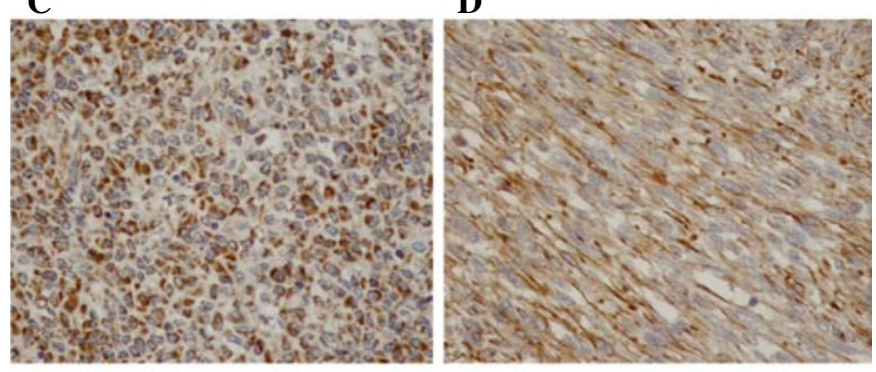

WT1-mutated

$\mathbf{E}$

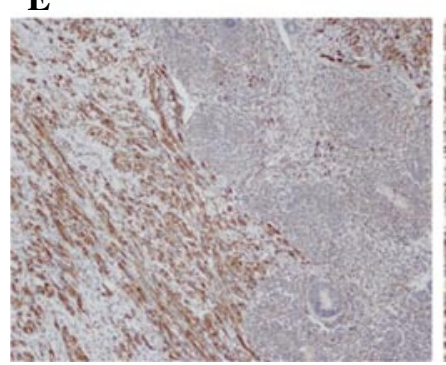

F

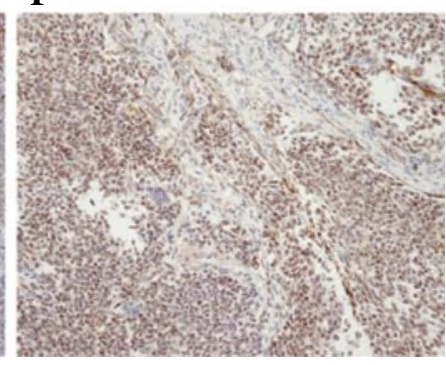

Figure 2. WT1 immunohistochemistry in nephroblastoma with wild-type WT1. (A) Positive staining in normal kidney tissue is limited to the glomerular epithelium. (B) In the tumor area, positive cytoplasmic and nuclear staining appears in the glomerular epithelium and blastema, but not in the collecting tubular epithelium. (C) Positive cytoplasmic immunoreactivity in blastema. (D) The stromal immunoreactivity in the majority of cases is limited to the cytoplasm (magnification, x40). (E and F) WT1 immunoreactivity in nephroblastomas with mutated WT1: (E) weak immunoreactivity appears in the stromal area in 2/4 cases; (F) blastemal staining in 1 case (magnification, $\mathrm{x} 20$ ).

intensities in the nephroblastomas. Staining was limited to the glomerular epithelium in the normal kidney tissue. Only light positive staining was found in the CCSK and PRCC samples. In cases with wild-type WT1, diffuse immunoreactivity was detected in the cytoplasm of the stromal and blastemal compo- 
A

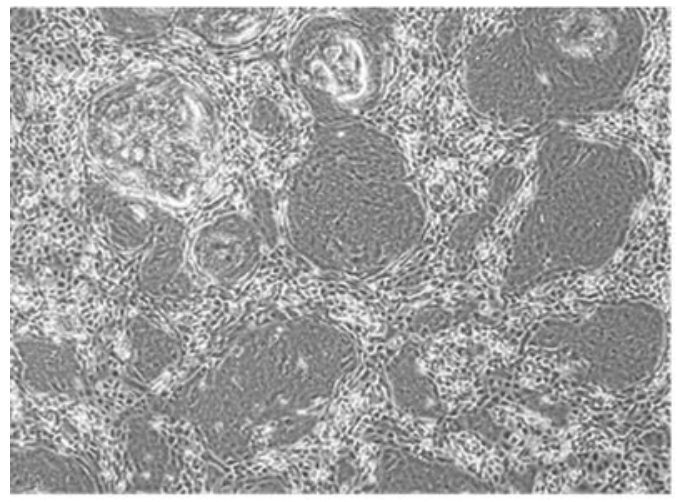

B

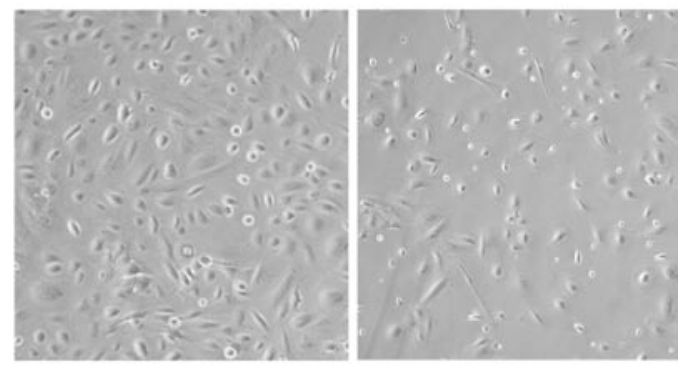

D
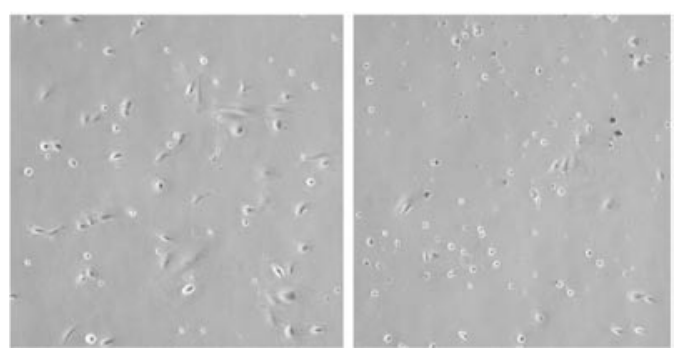

Figure 3. Primary culture of nephroblastoma from a case of recurrent disease (A) Cells in the first passage show arrangement in a glomerulous pattern. (B) Morphology of the cells in the third passage, immediately before a subculture for the transfection experiments. (C-E) Cells at the fourth passage at $70 \mathrm{~h}$ of transfection: (C) no treatment; (D) mock transfection (non-specific

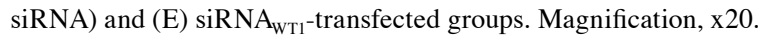

nents, sparing the tubular epithelium. Immunoreactivity in the cases with a $W T 1$ mutation was generally weaker and was confined to the stromal elements in 3 cases and detectable in the blastemal component in 1 case (WT20) (Fig. 2).

The study showed nuclear accumulation of $\beta$-catenin in the 4 cases of nephroblastoma harboring WT1 mutations, regardless of the $\beta$-catenin mutation status (data not shown). The positive nuclear-stained cells co-localized with WT1 immunoreactive cells. In the case with a point mutation of WT1, for which the WT1 immunohistochemistry was positive at the blastema, $\beta$-catenin nuclear staining was also positive in the same component. Membraneous staining of $\beta$-catenin was also detected in the glomerular epithelium of adjacent non-tumor tissue.

WT1 suppression inhibited the growth of nephroblastoma cells through increased apoptosis. The morphology of the primary nephroblastoma culture is shown in Fig. 3. In the siRNA $A_{\text {WT1 }}$ transfection experiment, the siRNA $\mathrm{WT}_{\mathrm{WT}}$ caused a reduction in WT1 expression (Fig. 4A). The siRNA ${ }_{\mathrm{WT} 1}$-transfected cells had a significantly slower growth from the 2 nd to the 4 th day
A

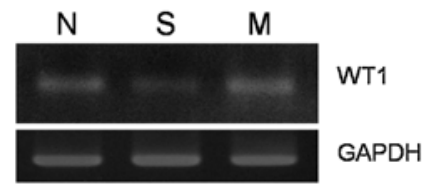

B

$\mathrm{X} 10^{3}$ cells/well

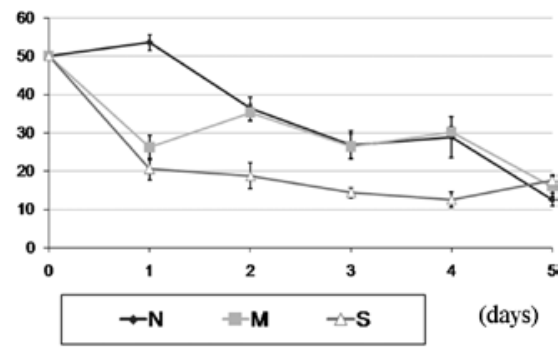

C

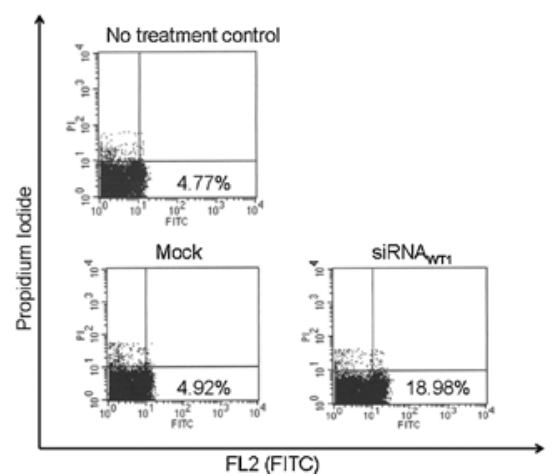

Figure 4. (A) RT-PCR results show a reduction in WT1 expression following siRNA $\mathrm{WT}_{\mathrm{WT}}$ transfection. (B) Cell survival after siRNA $\mathrm{WT}_{\mathrm{WT}}$ transfection. (C) Flow cytometric analysis of cells doubly stained with propidium iodide and Annexin-V. N, no treatment control; M, mock transfection and $\mathrm{S}$, siRNA $\mathrm{WT1}_{\mathrm{WT}}$-transfected groups.

of transfection, as compared to the mock transfection and no treatment groups (Fig. 4B). The apoptosis assay showed an increased apoptosis in the experimental group of 3.8 times the mock control (Fig. 4C).

\section{Discussion}

WT1 is a transcription factor that plays a role in genitourinary organ development. In pediatric nephroblastoma, the tumor suppressor role of $W T 1$ is supported by the classical evidence of mutations found in 5-10\% of tumors (5). However, the role of the gene in the subgroup of nephroblastomas that harbors the wild-type form of WTl has yet to be elucidated. An oncogenic role of WT1 in various human cancers suggests the possibility of its involvement in the development of nephroblastomas. Data from functional studies of WT1 in nephroblastomas are, however, limited as the tumor has only a limited number of cell lines available.

Our data from the qRT-PCR study showed that the average expression of WTI at the mRNA level in our pediatric nephroblastomas was significantly higher than that in the non-tumorous renal tissues. Data indicated that this 
overexpression was higher than the physiologic expression in the normal kidney tissue. When the expression level in the nephroblastoma tissues was compared to other pediatric renal tumors and neuroblastomas, the high level was found to be exclusive in the nephroblastomas, suggesting that WTI is associated with the tumorigenesis of this tumor. We used $\beta$-catenin, a known key player in pediatric embryonal tumors, as an external control and found a comparable expression of this gene in various tissues studied. Varying expression levels of $\beta$-catenin in the neuroblastomas may be explained by variations in tumor differentiation (12).

The total levels of WT1 in WT1 wild-type and WT1-mutated tumors were not different at the mRNA level. However, expression at the protein level in the 4 tumors with a WTI mutation studied showed a weaker intensity. The localization of WT1 proteins in nephroblastoma tissues were examined and the proteins were found to express in the blastemal and epithelial components of the tumors harboring wild-type WT1. Additionally, the proteins were confined to the stromal component in the WT1-mutated tumors, a difference that suggests different roles of $W T 1$ in the two nephroblastoma subclasses. This speculation is consistent with recent RNA microarray studies that found a difference in molecular signatures between nephroblastomas with and without a WT1 mutation $(4,13,14)$.

Fukuzawa et al described the expression pattern of WT1 in fetal kidney tissue (15). These authors found positive WT1 nuclear immunohistochemistry mostly in glomerular epithelium and blastema tissues. In our WT1 wild-type tumors, the localization patterns were comparable with those reported in developing kidneys (15). We speculated that WT1 marks an immature renal lineage in the tumor and may contribute to the growth of nephroblastoma tumor cells derived from developmentally arrested renal tissue.

We further tested the consequence of suppressing WT1 expression on nephroblastoma cell growth. Using a primary nephroblastoma culture, we created a WT1 knock-down model by using siRNA ${ }_{W T 1}$ transfection. The study found a negative growth effect on the siRNA $\mathrm{WT1}_{1}$-transfected group, suggesting an oncogenic role of the gene in this tumor subclass. The rapid reduction of the cells in the mock transfection and siRNA $A_{\mathrm{WT} 1}$ groups on the first day was explained by cytotoxicity caused by the transfection complexes. Additional evaluation of apoptotic activity showed increased apoptosis in the siRNA ${ }_{\mathrm{WT1}}$-treated cells. These results were consistent with previous evidence suggesting an anti-apoptotic role for WT1 (16).

An advantage of using cells cultured from ascites was that the culture was predominantly composed of tumor cells with a negligible amount of contaminating fibroblasts. However, the cells used were obtained from a patient undergoing chemotherapy. Therefore, the cells may not have exhibited a native nephroblastoma phenotype. The cells were from a single patient and may not have represented the complete range of possibilities from this subclass of WT1 wild-type nephroblastomas. Moreover, WT1 has at least four isoforms with different functions (16). Manipulation of its expression may also alter the isoform composition. Further studies focusing on the role of WT1 in this nephroblastoma subclass may be beneficial, not only for understanding the tumor biology, but also for possibly suggesting opportunities for the use of WT1 as a therapeutic target in this specific subset of tumor.
In conclusion, this study examined the mutation and expression of the WT1 gene in pediatric nephroblastomas and found a significantly high expression at the mRNA level, irrespective of the mutation status. At the protein level, differences in intensity and localization suggest its divergent roles in wild-type and mutated tumors. In the WT1 wild-type subclass of pediatric nephroblastomas, overexpression and negative growth effects on a suppressed expression suggest an oncogenic role of the gene in this tumor.

\section{Acknowledgements}

The authors thank Karnda Tongmitr of the Department of Pathology for the immunohistochemical studies. The apoptosis assays were performed at the Scientific Equipment Center, Prince of Songkla University. Dave Patterson edited the English language in the manuscript.

\section{References}

1. Beckwith JB, Kiviat NB and Bonadio JF: Nephrogenic rests, nephroblastomatosis, and the pathogenesis of Wilms' tumor. Pediatr Pathol 10: 1-36, 1990.

2. Li CM, Kim CE, Margolin AA, et al: CTNNB1 mutations and overexpression of Wnt/beta-catenin target genes in WT1-mutant Wilms' tumors. Am J Pathol 165: 1943-1953, 2004.

3. Rivera MN, Kim WJ, Wells J, et al: An X chromosome gene, WTX, is commonly inactivated in Wilms tumor. Science 315: 642-645, 2007.

4. Perotti D, Gamba B, Sardella M, et al: Functional inactivation of the WTX gene is not a frequent event in Wilms' tumors. Oncogene 27: 4625-4632, 2008.

5. Lee SB and Haber DA: Wilms tumor and the WT1 gene. Exp Cell Res 264: 74-99, 2001.

6. Moore AW, McInnes L, Kreidberg J, Hastie ND and Schedl A: YAC complementation shows a requirement for WT1 in the development of epicardium, adrenal gland and throughout nephrogenesis. Development 126: 1845-1857, 1999.

7. Sangkhathat S, Chotsampancharaen T, Kayasut K, Patrapinyokul S, Chiengkriwate P, Kitichet R and Maipang M: Outcomes of pediatric nephroblastoma in southern Thailand. Asian Pac J Cancer Prev 9: 643-647, 2008.

8. Oji Y, Yamamoto H, Nomura M, et al: Overexpression of the Wilms' tumor gene WT1 in colorectal adenocarcinoma. Cancer Sci 94: 712-717, 2003.

9. Sangkhathat S, Kusafuka T, Miao J, et al: In vitro RNA interference against $\beta$-catenin inhibits the proliferation of pediatric hepatic tumors. Int J Oncol 28: 715-722, 2006.

10. Wanitsuwan W, Kanngurn S, Boonpipattanapong T, Sangthong R and Sangkhathat S: Overall expression of beta-catenin outperforms its nuclear accumulation in predicting outcomes of colorectal cancers. World J Gastroenterol 14: 6052-6059, 2008.

11. Navakanit R, Graidist P, Leeanansaksiri W and Dechsukum C: Growth inhibition of breast cancer cell line MCF-7 by siRNA silencing of Wilms tumor 1 gene. J Med Assoc Thai 90: 2416-2421, 2007.

12. Sangkhathat $S$, Nara K, Kusafuka T, Yoneda A and Fukuzawa M: Artificially accumulated $\beta$-catenin inhibits proliferation and induces neurite extension of neuroblastoma cell line NB-1 via up-regulation of trkA. Oncol Rep 16: 1197-1203, 2006.

13. Fukuzawa R, Anaka MR, Weeks RJ, Morison IM and Reeve AE: Canonical WNT signaling determines lineage specificity in Wilms tumour. Oncogene 28: 1063-1075, 2009.

14. Fukuzawa R, Heathcott RW, More HE and Reeve AE: Sequential WT1 and CTNNB1 mutations and alterations of beta-catenin localisation in intralobar nephrogenic rests and associated Wilms tumours: two case studies. J Clin Pathol 60: 1013-1016, 2007.

15. Fukuzawa R, Heathcott RW, Sano M, Morison IM, Yun K and Reeve AE: Myogenesis in Wilms' tumors is associated with mutations of the WT1 gene and activation of Bcl-2 and the Wnt signaling pathway. Pediatr Dev Pathol 7:125-137, 2004.

16. Hohenstein P and Hastie ND: The many facets of the Wilms tumor gene, WT1. Hum Mol Genet 15: R196-R201, 2006. 